\title{
SARS-CoV-2 in a Neonate with Truncus Arteriosus: Management and Surgical Correction Timing
}

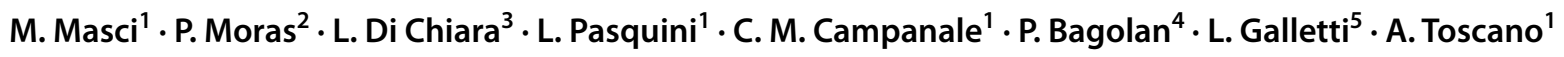

Received: 29 April 2021 / Accepted: 9 October 2021 / Published online: 21 October 2021

(c) The Author(s), under exclusive licence to Springer Science+Business Media, LLC, part of Springer Nature 2021

\begin{abstract}
Children seem to be less affected by SARS-CoV-2 infection. High risk categories should include patients with Congenital Heart Disease (CHD), both children and adults. We describe the case of a newborn with a postnatal diagnosis of Truncus Arteriosus (TA) type A1 without 22.q.11 deletion syndrome. Soon after birth, SARS-CoV-2 infection was transmitted by the father. Due to the onset of heart failure symptoms, diuretic therapy has been set up. For worsening of clinical conditions, inotropic support with milrinone was added. A progressive reduction of $\mathrm{N}$-terminal-pro hormone BNP over the days has been observed. Fourteen days after the negativization of the nasopharyngeal swab, the patient underwent surgical repair with Cardiopulmonary Bypass (CPB). Postoperative course was not complicated and the patient was discharged in good clinical conditions. There is very little evidence suggesting the optimal timing for surgery in SARS-CoV-2 positive patients. With a lack of specific guidelines, current strategy suggests a symptom-based or a polymerase chain reaction (PCR) test-based approach. In our case it was challenging to determine COVID-19 impact on heart failure symptoms. Our case is the first describing the surgical correction of CHD in a 40 days year old patient, performed in CPB after 14 days from SARS-CoV-2 infection negativization.
\end{abstract}

Keywords SARS-CoV-2 $\cdot$ Neonate $\cdot$ Congenital heart disease $\cdot$ Management $\cdot$ Surgical correction

$\begin{array}{ll}\text { Abbreviations } \\ \text { SARS-CoV-2 } & \begin{array}{l}\text { Severe Acute Respiratory Syndrome } \\ \text { Coronavirus 2 }\end{array} \\ \text { COVID-19 } & \text { Coronavirus disease 2019 } \\ \text { CHD } & \text { Congenital heart disease } \\ \text { TA } & \text { Truncus arteriosus } \\ \text { NICU } & \text { Neonatal intensive care unit }\end{array}$

M. Masci

marco.masci@opbg.net

1 Perinatal Cardiology Unit, Department of Medical and Surgical for Fetus-Neonate, Bambino Gesù Children's Hospital, IRCCS, Rome, Italy

2 Pediatric Department, University of Tor Vergata, Rome, Italy

3 Pediatric Cardiac Intensive Care Unit, Department of Pediatric Cardiology and Cardiac Surgery, Bambino Gesù Children's Hospital, IRCCS, Rome, Italy

4 Neonatal Surgery Unit, Department of Medical and Surgical for Fetus-Neonate, Bambino Gesù Children's Hospital, IRCCS, Rome, Italy

5 Pediatric Cardiac Surgery Unit, Department of Pediatric Cardiology and Cardiac Surgery, Bambino Gesù Children's Hospital, IRCCS, Rome, Italy

\author{
NT-proBNP N-terminal-pro hormone BNP \\ CBP Cardiopulmonary bypass \\ CDC Centers for disease control and prevention \\ PCR Polymerase chain reaction
}

\section{Introduction}

A novel coronavirus named Severe Acute Respiratory Syndrome Coronavirus 2 (SARS-CoV-2), causing coronavirus disease 2019 (COVID-19), has spread on a global scale from December 2019. Compared to adults, children seem to be less affected by SARS-CoV-2 infection, being usually asymptomatic or mildly symptomatic $[1,2]$. The reason might be found in the minor activity of ACE2 (the binding protein for SARS-CoV-2) in the pediatric population [3].

There is currently no evidence for intrauterine infection caused by vertical transmission in women who develop COVID-19 pneumonia in late pregnancy [4]. Perinatal transmission of COVID-19 is unlikely if correct hygiene precautions are undertaken and neonates who test positive for SARS-CoV-2 are largely asymptomatic or have mild self-limiting symptoms $[5,6]$. High risk categories should 
include patients with Congenital Heart Disease (CHD), both children and adults [7]. In a case series of 3 hospitalized children with pre-existing CHD (including five patients between 3 and 9 months), they had worsening of heart failure with increased BNP levels and required milrinone therapy. A new onset of cardiac arrhythmias has also been observed [8].

\section{Case Description}

We describe the case of a female newborn with a postnatally diagnosis of Truncus Arteriosus (TA). She was born at term (41 weeks of gestational age) from eutocic delivery with a weight of $3100 \mathrm{~kg}\left(25^{\circ}-50^{\circ}\right.$ percentile), length $50 \mathrm{~cm}$ $\left(50^{\circ}\right.$ percentile), head circumference $34 \mathrm{~cm}\left(50^{\circ}\right.$ percentile). APGAR score was 9-10. At first chest x-ray no pulmonary opacities or signs of pulmonary overload volume were found. On day 3 of life, she was transferred to the Neonatal Intensive Care Unit (NICU) of our hospital because of desaturation to $\mathrm{SpO} 288 \%$, tachypnea, dyspnoea and heart murmur presented in the first hours of life (Fig. 1).

Ultrasound confirmed type A1 Van Praagh TA, with the main pulmonary artery arising from the common trunk prior to giving off the left and right pulmonary arteries [9]. The truncal valve was tricuspid with mild to moderate regurgitation. Coronary arteries origins were high in the sinus of Valsalva: the left coronary artery had an initial intramural course, whereas the right one had a paracommissural origin. Genetic test for 22.q.11 deletion syndrome was negative.

During the 4th day of life, after the fallen of pulmonary vascular resistance, the patient started to present clinical signs of pulmonary over circulation with tachypnea and diaphragmatic retractions, tachycardia and bilateral hilar congestion on chest X-ray. Diuretic therapy with furosemide and high flow nasal cannula were started.

The SARS-CoV-2 nasopharyngeal swabs performed at the admission and after $48 \mathrm{~h}$ were negative. On the 9 th day of life, the infant tested positive for SARS-CoV-2. The infection was transmitted by the father immediately after the delivery. Therefore, the neonate was isolated in a negative pressure room. During the following days, due to increasing tachycardia associated with tachypnea, dyspnea and poor growth, diuretic therapy was set up. Due to the instability of the peripheral venous access, a central venous catheter was placed on the 22 nd day of life. For worsening of heart failure due to pulmonary overflow and failure to thrive, inotropic support with milrinone was commenced and therapy with diuretics was uptrited, with subsequent improvement of clinical conditions. N-terminal-pro hormone BNP (NT-proBNP) during the preoperative period was tested: with the optimization of heart failure therapy, a progressive reduction over the days was observed (Fig. 2).

The day before the surgery, a CT chest scan was performed for a better evaluation of parenchymal lung. The exam highlighted disventilative streaks in the middle lobe, faint subpleural interstitial thickening in the posterior area of haemodynamic significance. Besides, it has been described diffuse ectasia of the pulmonary vessels, due to overflow.

Fourteen days after the negativization of the nasopharyngeal swab, the patient underwent surgical repair with Cardiopulmonary Bypass (CPB). The correction included PAs removal from the arterial trunk, VSD closure and allograft valved conduit (Contegra $\mathrm{n}^{\circ} 12$ ) implantation between the right ventricle and the pulmonary artery. The weaning from $\mathrm{CPB}$ was supported with epinephrine, milrinone and vasopressin. In the Pediatric Cardiac Intensive Care Unit, nitric oxide was preemptively initiated based on the risk that the long preoperative period of pulmonary over circulation could have increased the risk of acute postoperative pulmonary vasoreactivity with progression to pulmonary hypertensive crises.

The patient was extubated on the 4th post-operative day and over the next days she resumed eating. The inotropic support was progressively suspended. On the 9th
Fig. 1 Chest X-rays showing cardiomegaly, hilar congestion and peribronchovascular thickening on preoperative on the 4 th day of life (A) and on the 20th day of life $(\mathbf{B})$
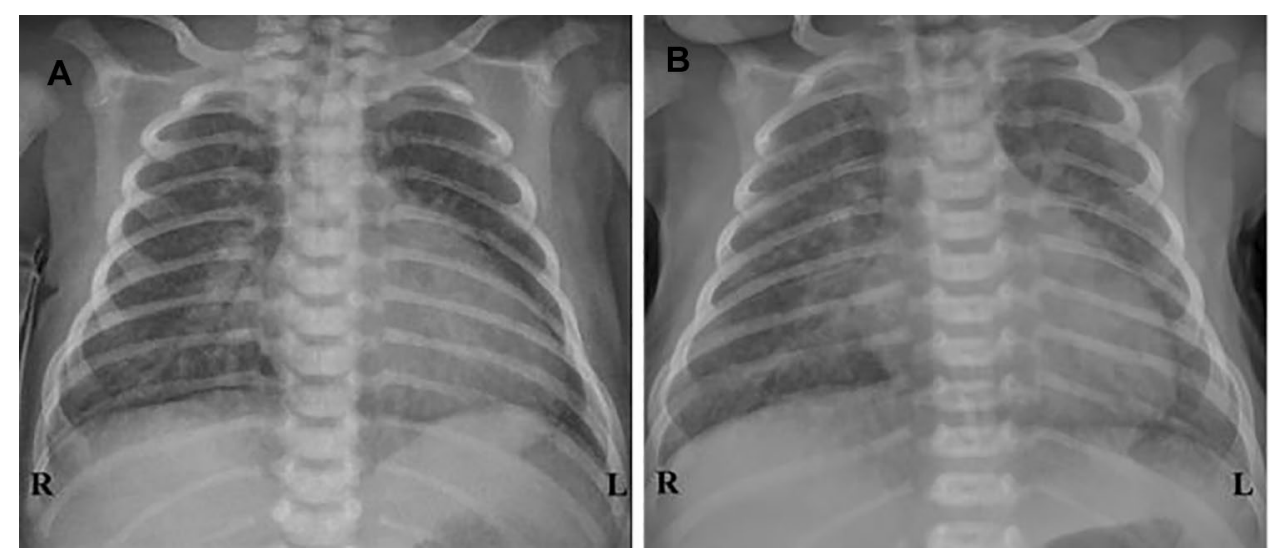
Fig. 2 Preoperative NTproBNB trend

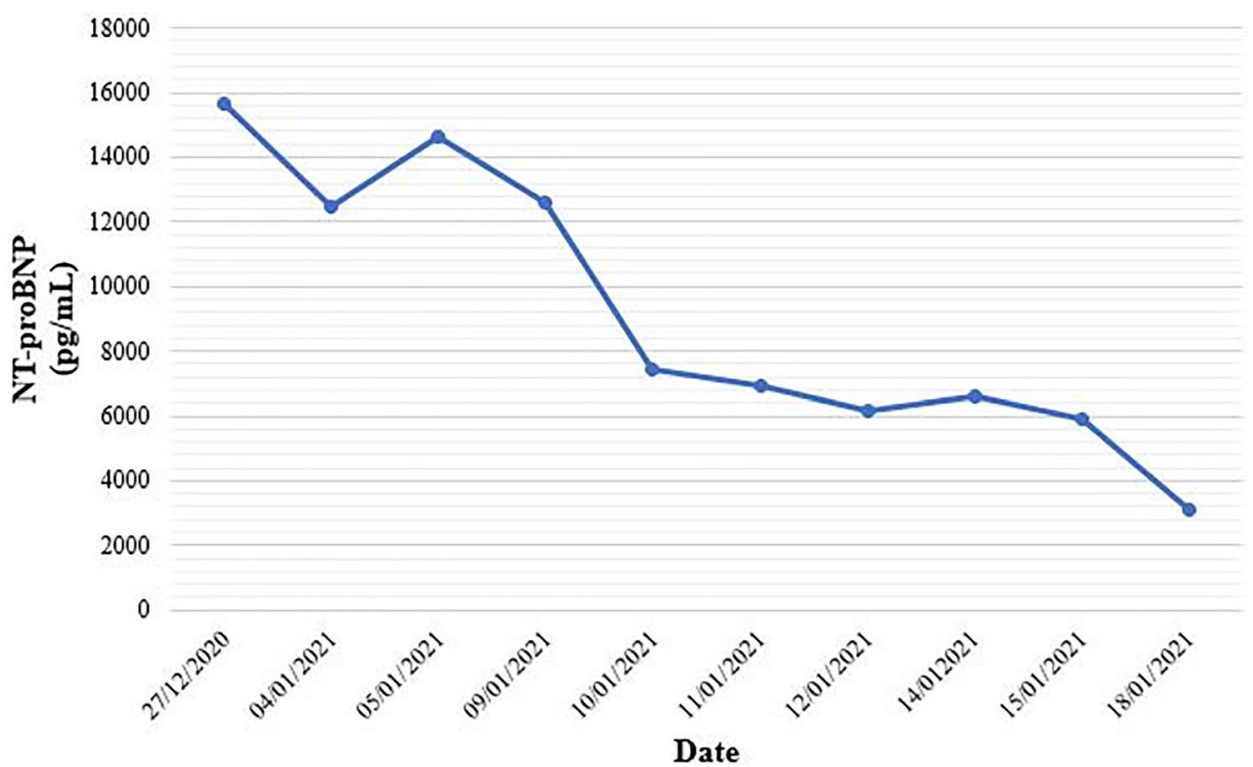

post-operative day, she was transferred to the Pediatric Cardiology ward and discharged in good clinical conditions after 10 more days.

\section{Discussion and Conclusion}

The present report is among the first to describe COVID-19 infection in a neonate with a severe CHD. To our knowledge, this is the first case in Italy describing the surgical correction and perioperative management of CHD in a neonate who tested positive for SARS-CoV-2. There is only one similar case described worldwide: a Blalock-Taussig shunt placement in a 15 days Sars-CoV2 positive neonate affected by Tetralogy of Fallot, which did not require CPB $[10,11]$.

The role of COVID-19 in worsening heart failure is difficult to assess. In our case, we are not sure that COVID19 worsened the underlying heart disease as cardiac heart failure is expected in neonates with TA after 25 days of life. Moreover, with a COVID-19-associated chest X-ray, abnormalities may be similar to those found in CHD with increased pulmonary blood flow (Fig. 3).

Crisis management strategies for congenital heart disease have recently been published. The document is not meant to be a guideline, but is designed to provide guidance for decision making [12].

There is very little evidence suggesting the optimal timing for surgery in SARS-CoV-2 positive patients. Choices should be individualized, considering both patient and provider safety. A symptom-based strategy is recommended by the Centers for Disease Control and Prevention (CDC). When symptom-based clearance is difficult (for instance, when the patient is asymptomatic, which appears more

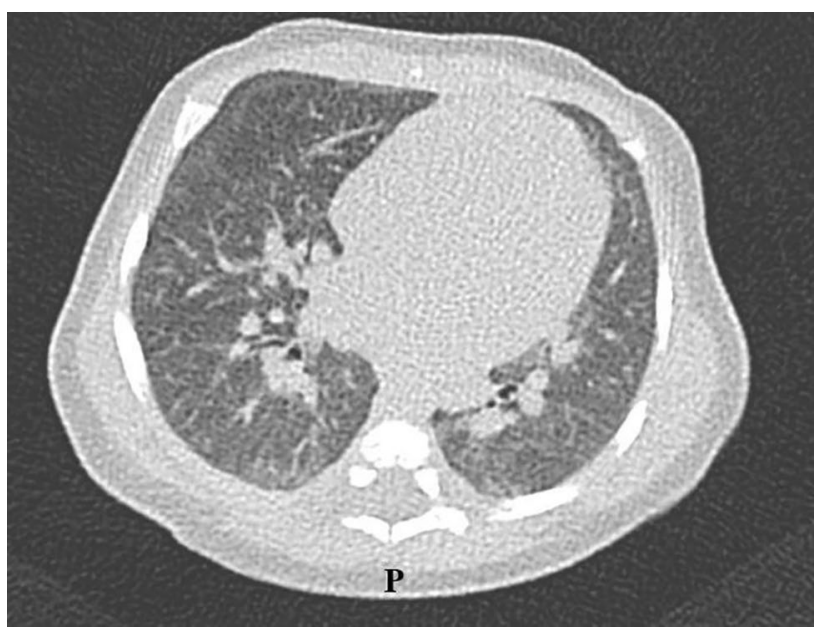

Fig. 3 The CT scan highlighted disventilative streaks in the middle Lobe; faint subpleural interstitial thickening in the posterior area of haemodynamic significance; diffuse ectasia of the pulmonary vessels, due to overflow

common in children as compared to adults), a polymerase chain reaction (PCR) test-based strategy may be considered. If prudent, surgery should be delayed until the patient's symptoms have improved and/or testing has been repeated (often after 14 days) and is negative $[13,14]$. Therefore, in this period, cooperation between pediatric cardiologists, cardio-anesthesiologists and cardiac surgeons is even more important to evaluate the clinical status of the patient and the risk of delaying surgery.

Certainly our case is the first describing the surgical correction of CHD in a 40 days patient, performed in CPB after 
14 days from SARS-CoV-2 infection negativization, without complication and a good early outcome.

Author Contributions MM and PM conceptualized and designed the study, drafted the initial manuscript, collected data, carried out the initial analyses and reviewed and revised the manuscript. DC, CMC, $\mathrm{PB}$ and LG designed the data collection instruments, analysis and interpretation of data, and reviewed and revised the manuscript. AT conceptualized and designed the study, coordinated and supervised data collection, and critically reviewed the manuscript for important intellectual content. All authors approved the final manuscript as submitted and agree to be accountable for all aspects of the work.

Funding No funding was secured for this study.

\section{Declarations}

Conflict of interest The other authors have no example conflicts of interest to disclose.

Ethical Approval Considering the nature of the analysis, the case report did not require the approval of the local ethics committee according to current legislation, but a notification was sent.

\section{References}

1. Huang C, Wang Y, Li X et al (2020) Clinical features of patients infected with 2019 novel coronavirus in Wuhan. China Lancet 395:497-506

2. Hong W, Wang Y, Chun HT et al (2020) Clinical characteristics of novel coronavirus disease 2019 (COVID-19) in newborns, infants and children. Pediatr Neonatol 61(2):131-132

3. Dong Y, Mo X, Hu Y et al (2020) Epidemiological characteristics of 2143 pediatric patients with 2019 coronavirus disease in China. Pediatrics 145(6):e20200702
4. Chen H, Guo J, Wang C et al (2020) Clinical characteristics and intrauterine vertical transmission potential of COVID-19 infection in nine pregnant women: a retrospective review of medical records. Lancet 395(10226):809-815

5. Salvatore CM, Han J-Y, Acker KP et al (2020) Neonatal management and outcomes during the COVID-19 pandemic: an observation cohort study. Lancet Child Adolesc Health 4(10):721-727

6. Zeng L, Xia S, Yuan W et al (2020) Neonatal early-onset infection with SARS-CoV-2 in 33 neonates born to mothers with COVID19 in Wuhan, China. JAMA Pediatr 174(7):722-725

7. Tan W, Aboulhosn J (2020) The cardiovascular burden of coronavirus disease 2019 (COVID-19) with a focus on congenital heart disease. Int J Cardiol 309:70-77

8. Simpson M, Collins C, Nash DB et al (2020) Coronavirus disease 2019 infection in children with pre-existing heart disease. J Pediatr 227:302-307

9. Van Praagh R, Van Praagh S (1965) The anatomy of common aorticopulmonary trunk (truncus arteriosus communis) and its embryologic implications. A study of 57 necropsy cases. Am J Cardiol 16:406-425

10. Giamberti A, Varrica A, Agati S et al (2020) Impact of the coronavirus disease 2019 (COVID-19) pandemic on the Italian congenital cardiac surgery system: a national survey. Eur J Cardiothorac Surg 58:1254-1260

11. Salik I, Mehta B (2020) Tetralogy of Fallot palliation in a Covid19 positive neonate. J Clin Anesth 66:109914

12. Stephens EH, Dearani JA, Guleserian KJ et al (2020) COVID-19: crisis management in congenital heart surgery. Ann Thorac Surg 110(2):701-706

13. https://www.cdc.gov/coronavirus/2019-ncov/hcp/dispositionhospitalized-patients.html. Accessed 17 April 2020

14. Levy E, Blumenthal J, Chiotos K et al (2020) COVID-19 FAQs in pediatric cardiac surgery. World J Pediatr Congenit Heart Surg 11(4):485-487

Publisher's Note Springer Nature remains neutral with regard to jurisdictional claims in published maps and institutional affiliations. 